\title{
Das Journal of Intellectual Property, Information Technology and Electronic Commerce Law (JIPITEC)
}

Thomas Dreier/Gerald Spindler/Philipp Schmechel*
A. Einleitung
136
B. Gründung im Jahr 2009 und zweite
Förderphase.
C. Organisationsstruktur ................ 139
I. Herausgeber
II. Korrespondentennetzwerk
III. Zentrale Koordinierung
140

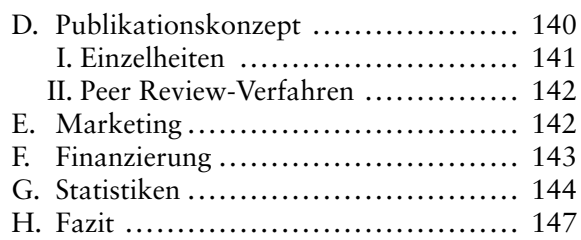

140

Der Beitrag stellt die kostenfreie internationale Open Access-Zeitschrift „Journal of Intellectual Property, Information Technology and Electronic Commerce Law" (JIPITEC) vor. Die vor zehn Jahren gegründete Fachzeitschrift bietet eine Plattform für vor allem auch längere wissenschaftliche Beiträge zu allen Themen des europäischen Immaterialgüter- und Internetrecht in vornehmlich englischer Sprache. JIPITEC hat sich mittlerweile als eine der wenigen juristischen Open-AccessZeitschriften in Europa etabliert und veröffentlicht Aufsätze renommierter Rechtswissenschaftler. Der Artikel gibt einen Überblick über den Entstehungsprozess sowie über die Organisationsstruktur des Journals, insbesondere die Entwicklung der Herausgeberschaft, das Korrespondentennetzwerk sowie die zentrale Koordinierung der Hefterstellung. Zudem werden das Publikationskonzept von JIPITEC sowie Details des Peer Review-Verfahrens erläutert. Anschließend zeigt der Beitrag das Marketingkonzept und weitere Maßnahmen zur Erhöhung der Sichtbarkeit des Journals auf und beschreibt den schwierigen Faktor der Finanzierung einer kostenfreien, nicht-kommerziellen und rein aus Drittmitteln finanzierten Open AccessZeitschrift. Statistiken zur Herkunft der Leser sowie zu den Besucherzahlen bei JIPITEC runden den Artikel schließlich ab.

\section{A. Einleitung}

Auch wenn die Möglichkeiten, die Digitalisierung und Vernetzung angesichts der Publikationskrise (,journal crisis“) für die Open Access-Verbreitung wissenschaftlicher Informationen bieten, seit mehr als zwei Jahrzehnten diskutiert werden, ${ }^{1}$ ist vor allem die deutsche rechtswissenschaftliche Zeitschriftenlandschaft noch immer weitgehend durch proprietäre Geschäftsmodelle gekennzeichnet. Soweit Informa-

* Prof. Dr., Thomas Dreier, Institut für Informations- und Wirtschaftsrecht, Karlsruher Institut für Technologie (KIT); Prof. Dr., Gerald Spindler, Institut für Wirtschafts- und Medienrecht, Universität Göttingen; Philipp Schmechel, LL.M. (Sydney), Doktorand, Universität Göttingen.

1 S. nur etwa Shaw/Moore (Hg.), Electronic publishing in science, Paris 1996; Butterworth (Hg.), The Impact of Electronic Publishing on the Academic Community, London 1998; National Academies (Hg.), Electronic, Scientific, Technical, and Medial Journal Publishing and its Implications, Washington 2004 . 
tionen (auch) online angeboten werden, geschieht dies wie zuvor schon für Papierversionen nach wie vor ebenfalls auf der Grundlage von Abonnement-Modellen. Diese Situation findet sich auch im Bereich des Immaterialgüter-, Internet- und ECommerce-Rechts wieder. Da die Online-Angebote überdies eng an die Wiedergabe der Offline-Publikationen gebunden sind, bleibt zudem das Potential für längere Artikel unausgeschöpft. Aus diesem Grund war die Publikationslandschaft auf dem Gebiet des Immaterialgüter- und Internetrechts durch eine Reihe kostenpflichtiger deutschsprachiger Angebote gekennzeichnet, die durch einige englische wie vor allem US-amerikanische Zeitschriften ergänzt wurden, die durchaus auch längeren akademischen Studien Raum boten. Darüber hinaus fehlte es an einer Zeitschrift, die nach dem Muster einiger - allerdings ebenfalls hochkommerzieller, vor allem englischer - Zeitschriften auf dem Gebiet des geistigen Eigentums das Internetrecht aus europäischer Sicht in den Blick nahm.

Diese Lücken für das Recht des geistigen Eigentums und das Internetrecht zu schließen hat sich das „Journal of Intellectual Property, Information Technology and Electronic Commerce Law" (JIPITEC) vorgenommen (www.jipitec.eu). Es handelt sich um ein kostenfreies Open Access Journal mit einem „double blind“ Peer Review-Verfahren, das auch von den Autoren keine Gebühren (sog. „article processing charges“, APC) verlangt, sondern sich stattdessen ohne Gewinnerzielung rein aus Drittmitteln finanziert. Inhaltlich bietet JIPITEC eine Plattform für vor allem auch längere wissenschaftliche Beiträge zu allen Themen des europäischen Immaterialgüter- und Internetrecht in vornehmlich englischer Sprache, ohne jedoch auf diese eine Sprache beschränkt zu sein. Geleitet wird JIPITEC durch ein Gremium von Herausgebern, die sich aus unterschiedlichen EU-Mitgliedstaaten rekrutieren. Die traditionelle, von Print-Zeitschriften „ererbte“ Struktur von jährlich drei Heften bzw. Ausgaben, für die reihum jeweils ein anderer Herausgeber/eine andere Herausgeberin verantwortlich zeichnet, wurde allerdings auch in der Online-Umgebung beibehalten.

Im zehnten Jahr nach dem Erscheinen des ersten Heftes hat sich JIPITEC gut entwickelt. Die Sichtbarkeit ist kontinuierlich gestiegen, ${ }^{2}$ zugleich ist die Zeitschrift für eine zunehmende Zahl von Autoren aus ganz Europa zur Präsentation ihrer Forschungsergebnisse interessant, auch wenn JIPITEC nicht mit dem gleichen Impact-Factor aufwarten kann, wie manch andere renommierte Zeitschrift vor allem im englischsprachigen Ausland. Zugleich wird dadurch der innereuropäische Austausch auf den genannten Rechtsgebieten gefördert. Schließlich können deutsche Autoren, deren Aufsätze in deutschsprachigen Zeitschriften auf europäischer Ebene nur beschränkt rezipiert werden, nun auch außerhalb Deutschlands und der nationalen Fach-Community wahrgenommen werden.

2 S. die Aufschlüsselung der Zugriffszahlen unter G. 


\section{B. Gründung im Jahr 2009 und zweite Förderphase}

Die Idee für eine Open Access Zeitschrift auf dem Gebiet des Immaterialgüter-, des Internet- sowie des Rechts des E-Commerce entstand in Zusammenarbeit der drei Professoren Gerald Spindler (Göttingen), Axel Metzger (Berlin) und Thomas Dreier (Karlsruhe) als Antwort auf das LIS-Förderprogramm „Wissenschaftliche Zeitschriften " der Deutschen Forschungsgemeinschaft. ${ }^{3}$ Dieses Programm zielte auf die Förderung von Infrastrukturen ab, in denen technische oder organisatorische Innovationen im Bereich elektronischer Publikationen angeschoben sowie neue Geschäftsmodelle entwickelt und erprobt werden können. ${ }^{4}$

Nachdem der DFG-Antrag in einer ersten Phase für drei Jahre mit einer Fördersumme von $39.500 €$ zuzüglich einer Programmpauschale i.H.v.7.900€ unter Hinweis auf die Bekanntheit der Antragsteller wie auch des „thematischen $\mathrm{Zu}$ schnitt[s], der zu einer wesentlichen Vertiefung des wissenschaftlichen Diskurses im Bereich des IT- und Urheberrechts" beizutragen vermag, bewilligt worden war, ${ }^{5}$ war vorrangige Aufgabe die Erstellung der technischen Infrastruktur (Serverumgebung; Webseiten-Design usw.). Hosting und Redaktionssoftware wurde zunächst im Rahmen einer eigens geschlossenen Kooperationsvereinbarung mit dem Hochschulbibliothekszentrum des Landes NRW sichergestellt.

Sozusagen als Startschuss machte sich JIPITEC dann mit einer Konferenz bekannt, die unter der Ägide von Prof. Metzger an der Universität Hannover zu den Themen Open Source, Commons, User, Service Provider mit Beiträgen internationaler Fachvertreter ausgerichtet wurde. Die Beiträge dieses Symposiums bildeten neben einem gemeinsamen Vorwort der Herausgeber zur Motivation der Zeitschrift zugleich den Grundstock für die erste Ausgabe der neuen Zeitschrift.

Nach den Vorarbeiten des Jahres 2009, stand das zweite Jahr im Zeichen der Veröffentlichung der weiteren Ausgaben, deren Umfang gegenüber der ersten Ausgabe dann um einiges zunahm. Auf Grund der kurzen Zeitspanne, die zwischen der Fertigstellung von Redaktionssystem und Website, so wie der Veröffentlichung der ersten Ausgabe lag, konnten viele Erfahrungen erst im laufenden Betrieb gewonnen werden. Diese umzusetzen und hierbei zugleich die Kontinuität zu wahren hat sich, insbesondere vor dem Hintergrund der hohen Präsenz in Fachkreisen, die JIPITEC von Anfang an genoss, als besonders anspruchsvoll herausgestellt. Angesichts des guten Starts, im Lichte jedoch der noch verbleibenden Aufgaben, bewilligte die

3 Projektantrag, Geschäftszeichen SP 587/6-1 und zum Folgeantrag SP 587/6-2. - Im Jahr 2015 wurde das Förderprogramm „Wissenschaftliche Zeitschriften“ in das Programm „Infrastruktur für elektronische Publikationen und digitale Wissenschaftskommunikation" überführt (s. www.dfg.de/foerderun g/info_wissenschaft/2015/info_wissenschaft_15_03/index.html).

$4 \mathrm{Zu}$ Einzelheiten der Finanzierung von JIPITEC s. noch nachfolgend unter F.

5 Anschreiben, Bewilligungsbescheid v. 16.2.2009. 
DFG im Jahr 2012 schließlich eine Verlängerung der Förderung um weitere drei Jahre im Weg einer weiteren Sachbeihilfe i.H.v. insgesamt 34.200€.

\section{Organisationsstruktur}

\section{Herausgeber}

Der Herausgeberkreis ist in den inzwischen zehn Jahren des Bestehens von JIPITEC kontinuierlich um Vertreter aus anderen EU-Mitgliedstaaten erweitert worden. Neben den bereits genannten Gründungsherausgebern Prof. Gerald Spindler (Universität Göttingen), Prof. Axel Metzger (inzwischen Humboldt-Universität Berlin) und Prof. Thomas Dreier (Karlsruher Institut für Technologie), gehören dem Kreis der Herausgeber die folgenden weiteren Personen an (in der Reihenfolge ihres Dazustoßens): Prof. Lucie Guibault, (inzwischen Dalhousie University, Halifax; seit 2011); Prof. Miquel Peguera Poch (Universitat Oberta de Catalunya; seit 2012); Prof. Séverine Dusollier (SciencesPo, Paris; seit 2015); Prof. Chris Reed (Queen Mary University, London; seit 2016) und Prof. Karin Sein (Universität Tartu; seit 2017). Auf diese Weise sind neben dem deutschsprachigen Raum sowohl die spanisch- und französischsprachigen Mitgliedstaaten der EU wie auch das Vereinigte Königreich und die baltischen Staaten abgedeckt.

Den Herausgebern obliegt in kollegialer Abstimmung die Fortentwicklung der strategischen Ausrichtung der Zeitschrift insgesamt. Zugleich fungiert jeder Herausgeber für jeweils ein Heft der JIPITEC als allein verantwortlicher Herausgeber. Seine Aufgabe reicht vom Einwerben der Beiträge und Buchbesprechungen, der Durchsicht der eingereichten Beiträge, über die Organisation des Peer Review-Prozesses und die Kommunikation mit den Autoren der eingereichten Beiträge bis hin zur Fertigstellung des jeweiligen Heftes. Dabei wird er von der zentralen Geschäftsstelle unterstützt, die vor allem für die Kommunikation mit dem englischsprachigen Copy-Editor, das Layout und schließlich die Online-Stellung des Heftes Sorge trägt.

\section{Korrespondentennetzwerk}

Neben den Herausgebern ist von Anbeginn an ein Netzwerk nationaler Korrespondenten geknüpft worden, um sowohl weitere Länder abzudecken als auch herausragende Wissenschaftler an die JIPITEC zu binden. ${ }^{6}$ Dazu zählen (in alphabetischer Reihenfolge): Prof. Dr. Graeme Dinwoodie, LL.M. (Harvard), Chicago-Kent College of Law; Prof. Dr. Ejan Mackaay LL.M., LL.D., (Amsterdam), Université de Montréal; Dr. Rita Matulionyte, LL.M (Munich), University of Newcastle; Prof. Dr. Christophe Geiger, Centre d'Études Internationales en Propriété Intellectuelle (CEIPI) Straßbourg; Prof. Dr. Giovanni M. Riccio, Università degli Studi di

6 www.jipitec.eu/about-the-journal/editorial-staff. 
Salerno; Prof. Dr. Cyrill P. Rigamonti, LL.M. (Georgetown), S.J.D. (Harvard), Universität Bern; Prof. Dr. Olav Torvund, Universitetet i Oslo; Prof. Dr. Mikko Välimäki, LL.M. (Helsinki), Helsinki University of Technology; Prof. Dr. Rolf H. Weber, Universität Zürich; Prof. Dr. Andreas Wiebe, LL.M. (Virginia), Georg-August-Universität Göttingen; sowie Prof. Dr. Raquel Xalabarder, LL.M. (Columbia), Universitat Oberta de Catalunya.

Die auch jenseits von JIPITEC in vielfältigem Kontakt stehenden Korrespondenten haben als Ideengeber und durch Marketing als Multiplikatoren wesentlich zum Erfolg von JIPITEC beigetragen. Sie weisen vor allem jüngere Autoren auf die Veröffentlichungsmöglichkeit in JIPITEC hin. Zugleich können sie die Veröffentlichung von „Special Issues“ anregen, in denen die Ergebnisse thematisch konzentrierter, herausragender Konferenzen veröffentlicht werden können.

\section{Zentrale Koordinierung}

Der ursprünglich verfolgte Ansatz, sämtliche mit der Erstellung eines Heftes verbundenen Aufgaben bis hin zur Organisation des „copy editing“ und des Layouts der einzelnen Beiträge jeweils am Lehrstuhl des für das betreffende Heft zuständigen Herausgebers anzusiedeln, hatte sich jedoch schon bald als nicht tragfähig erwiesen, da für das verwendete Textsatzprogramm (Adobe InDesign) für jeden Lehrstuhl eine eigene Lizenz hätte erworben und die einzelnen Mitarbeiter zugleich entsprechend in der Benutzung hätten instruiert werden müssen.

Fortan wurden die Aufgaben der Textgestaltung daher zentral von einem Mitarbeiter am Lehrstuhl von Prof. Spindler in Göttingen übernommen (zunächst Philipp Zimbehl, nachfolgend Philipp Schmechel). Das betrifft auch die Aufgaben des Tagesgeschäfts, also insbesondere die Pflege des E-Mail-Verteilers für die Kommunikation mit dem Korrespondentennetzwerk und für interessierte Leser wie auch die Pflege der Website und die zentrale Administration des Projekts sowie nicht zuletzt die Beantwortung von Anfragen und den Ausbau der weiteren Sichtbarkeit in sozialen Medien.

\section{Publikationskonzept}

Unter dem Blickwinkel von Open Access ist zentral ist jedoch das Publikationskonzept von JIPITEC. Für entscheidend haben die Gründungsherausgeber erachtet, dass JIPITEC ein peer-reviewtes Open Access Journal ist, das sowohl für Autoren wie für Nutzer kostenlos ist. ${ }^{7}$

7 Zur Finanzierung s. noch unten F. 


\section{Einzelheiten}

Im Einzelnen bedeutet das:

- Die Zeitschrift basiert primär auf einer Online-Publikation. Print-Ausgaben werden nicht erstellt, aber zum Selbstausdruck auf PDF-Basis zur Verfügung gestellt.

- Primäre Publikationsform ist die HTML-Datei, versehen mit Randnummern für Textabsätze, um die Zitierung zu erleichtern.

- Die Beiträge stehen sowohl einzeln, als auch als Gesamtausgabe gebündelt, zum Download bereit.

- Die Beiträge stehen unter einer speziellen, zunächst von Prof. Metzger für das Land Nordrhein-Westfalen entwickelten Lizenz (DPPL). Aufgrund von Problemen in der internationalen Akzeptanz ist JIPITEC inzwischen dazu übergegangen, die international bekannteren Lizenzen der Creative-Commons-Familie zu verwenden. Welche Lizenzform sie wählen wollen, steht den Autoren jedoch weitgehend frei, solange nur der Open Access-Charakter der Publikation gewahrt wird. Inzwischen entscheiden sich beinahe alle Autoren für eine der CCLizenzen. Eine spätere wirtschaftliche Verwertbarkeit der Ergebnisse ist insoweit nicht möglich, da es sich bei JIPITEC um eine reine non-profit Arbeit handelt.

- Nach längerer Diskussion haben sich die Herausgeber jedoch entschieden, die veröffentlichten Beiträge nicht mit einer Kommentar- oder gar einer Änderungsfunktion zu versehen. Grund für diese Entscheidung war die unsichere Rechtslage in Bezug auf die Haftung für Fremdbeiträge und der mit einer derartigen Funktion verbundene Kontroll- und Personalaufwand.

- Für die Beiträge wird weder eine Peer Review-Gebühr noch eine Publikationsgebühr oder sonstige Kosten erhoben. Das Journal ist für Autoren wie für Leser kostenfrei. Das schließt den Service einer (kostenlosen) sprachlichen Endkontrolle der zur Publikation angenommenen Artikel durch externe Native Speaker (sog. „copy-editing“) ebenso mit ein wie ein kostenloses Proof-Reading.

- Werbliche Hinweise finden sich in der Rubrik „Events“ zum einen auf aktuelle Veranstaltungen, insbesondere Tagungen aus dem IT-Recht, und zum anderen in der Rubrik „Jobs“ als aktuelle Stellenausschreibungen im akademischen Bereich sowie sonstigen Fällen, die für die Leserschaft von Relevanz sein könnten.

- Das Journal erscheint ähnlich anderen internationalen archiv-ähnlichen Zeitschriften in der Regel drei Mal im Jahr. Zugleich besteht jedoch die Möglichkeit der Herausgabe dazwischen geschobener Sonderausgaben (s. das von Axel Metzger mit Beiträgen einer von Prof. Heike Schweizer organisierten Konferenz zum Thema "Contracts on Digital Goods and Services”, herausgegebene JIPITEC Vol. 8 (1) 2017, sowie das von Martin Husovec herausgegebene Sonderheft JIPITEC Vol. 8 (3) 2017 zum Thema „Intermediary Liability as a Human Rights Issue”). 


\section{Peer Review-Verfahren}

Zur Qualitätssicherung der eingereichten Manuskripte dient neben der Expertise der jeweiligen Herausgeber der einzelnen Hefte insbesondere das Peer Review-Verfahren. Dieses Verfahren ist zwar bei deutschen rechtswissenschaftlichen Zeitschriften kaum verbreitet, bei denen die Qualitätskontrolle meist allein durch Verlagslektorate erfolgt. Mit der Entscheidung für ein Peer Review-Verfahren, das überdies „double blind“ ausgestaltet ist (es erfährt also weder der Reviewer den Namen des Autors, noch umgekehrt der Autor den Namen des Reviewers), übernimmt JIPITEC bewusst ein Qualitätssicherungsverfahren, wie es im anglo-amerikanischen Sprachraum üblich ist. In den die eingegangenen Manuskripte beurteilenden Reviews findet sich zumeist eine Reihe von Hinweisen, die nach Weiterleitung an den Autor zu einer Schärfung der Argumentation und einer Verbesserung der Qualität der eingereichten Manuskripte führt.

Die Übernahme der Reviews erfolgt durch ad hoc-Ansprache geeigneter Personen seitens des jeweiligen Heftherausgebers auf freiwilliger Basis. Ein Honorar wird nicht gezahlt. Dass dieses Ziel erreicht werden konnte, ist der großen Bereitschaft zur Mitarbeit unter den Reviewern und Reviewerinnen zu verdanken, ebenso wie den persönlichen Netzwerken der jeweiligen Herausgeber/innen. Angesichts dessen wurde bislang auch von einer Standardisierung von Evaluationsformularen und einer Automatisierung des Verfahrens, wie sie bei größeren Zeitschriften des Öfteren üblich ist, abgesehen.

Die Qualität der einzelnen Reviews ist ein unabdingbarer Grundstein für die Qualität der in JIPITEC veröffentlichten Beiträge, die ihrerseits wiederum Voraussetzung dafür ist, dass JIPITEC für Wissenschaftler als Online-Plattform für ihre Veröffentlichungen interessant ist.

\section{E. Marketing}

Die hohe Sichtbarkeit für das Publikum bildet die zentrale Legitimation einer Open Access-Veröffentlichung. Weiterhin wird die Zeitschrift durch eine große Sichtbarkeit für Autoren attraktiver. Da es erklärtes Ziel von JIPITEC ist, qualitativ hochwertige Beiträge besonders renommierter Autoren frei zugänglich anzubieten, galt es seit jeher, Autoren für eine Veröffentlichung in JIPITEC auch ohne Vergütung und unter freier Lizenz zu gewinnen. Dies ist nur bei entsprechend hoher Reputation der Zeitschrift zu erreichen. Um dem Charakter als reine Online-Zeitschrift ohne traditionelle Vertriebs- und Marketingstrukturen auf der einen und einem Themenspektrum, das nicht auf digitale Inhalte beschränkt ist, auf der anderen Seite gerecht zu werden, wurde eine Marketingstrategie verfolgt, die sich sowohl klassischer Instrumente bedient, als auch die Besonderheiten des Internets in Betracht zieht. 
So war JIPITEC mit klassischem Werbematerial auf Konferenzen wie den Göttinger Urheberrechtstagungen, oder den Open-Access-Tagen ebenso präsent wie in sozialen Medien (Twitter, LinkedIn). Ergänzt wird dies durch einen Email-Newsletter mit über 759 internationalen Abonnenten, der bezüglich jeder Ausgabe verschickt wird. Nicht zuletzt wird in enger Zusammenarbeit mit Bibliotheken und elektronischen Zeitschriftenverzeichnissen auf eine erhöhte Sichtbarkeit und Bekanntheit hingearbeitet. So konnten Listungen im Directory of Open Access Journals, im Gemeinsamen Verbundkatalog des GBV sowie in anderen regionalen und überregionalen Bibliothekskatalogen ebenso erreicht werden wie eine Listung bei HeinOnline (wo auch die Volltexte verfügbar sind) und bei Google Scholar. Die Verfügbarkeit bei Scopus soll in naher Zukunft erfolgen.

\section{F. Finanzierung}

Wie für viele Open Access-Zeitschriften, ist die Frage des Finanzierungsmodells zentral. Grundsätzlich stehen hier mehrere Möglichkeiten zur Verfügung. Die Ausgaben, die zur Bearbeitung der Artikel und zur Unterhaltung der Infrastruktur erforderlich sind, können entweder von den Autoren der veröffentlichten Artikel, von den Lesern der Open Access-Zeitschrift oder von der Zeitschrift selbst getragen werden. Denkbar ist auch eine Kostentragung durch mehrere oder alle der Genannten.

Bei der Entscheidung des Finanzierungsmodells, das JIPITEC zugrunde liegt, galt es vor allem, den bestehenden Besonderheiten des Publizierens im Bereich der Rechtswissenschaften Rechnung zu tragen. Da der Zugriff auf JIPITEC als Open Access Journal für die Nutzer dem Gedanken von Open Access entsprechend kostenfrei zugänglich sein sollte, schied eine Kostenbelastung der Nutzer von vorneherein aus. Da vor allem in Deutschland Autoren rechtswissenschaftlicher Fachaufsätze im traditionellen Printbereich nicht nur keine Veröffentlichungsgebühr bezahlen müssen, sondern umgekehrt von den Verlagen für die Einräumung der Rechte an ihren Artikeln entlohnt werden, hätte die Entscheidung für eine ArtikelGebühr seitens der Autoren (APC) für JIPITEC von vorne herein einen entscheidenden Wettbewerbsnachteil gegenüber den bereits etablierten rechtswissenschaftlichen Zeitschriften bedeutet. Daher schied also auch diese zweite Option aus. Die Finanzierung muss daher vom Journal selbst getragen werden. Das stellt für eine Zeitschrift, die sich strikt als nicht-kommerziell versteht und keine Werbung enthält, eine besondere Herausforderung dar.

Einen ersten Schritt zur erfolgreichen Bewältigung dieser Herausforderung stellte die Reduktion der Ausgaben auf ein Minimum dar. So arbeiten sämtliche Herausgeber, Korrespondenten und Peer Reviewer ehrenamtlich ohne Vergütung im Rahmen ihres „Dienstes an der Wissenschaft“. Die extern erbrachte Leistung des „co- 
py-editing “ hingegen wird finanziell angemessen vergütet. Zusammen mit den Ausgaben für die Infrastruktur konnten die erforderlichen laufenden Kosten in einer Größenordnung zwischen 5.000 und $6000 €$ pro Jahr gehalten werden.

Dieser Finanzierungsbedarf war in der Startphase von JIPITEC aufgrund der Förderung durch die Deutsche Forschungsgemeinschaft gewährleistet. Nach Ablauf der zweiten Förderphase war eine weitere Verlängerung jedoch nicht möglich, da die Mittel lediglich für den Anschub, nicht hingegen für den nachfolgenden Betrieb vorgesehen waren. Als wenig hilfreich erwies sich hier, dass die DFG gleichzeitig Mittel für Finanzierung von APCs auflegte, deren Inanspruchnahme für JIPITEC nicht in Betracht kam, da auf APCs aus den genannten Gründen verzichtet worden war.

Als Lösung entwickelten die Herausgeber von JIPITEC in Gesprächen mit dem Vorstand der Deutschen Gesellschaft für Informatik e.V. (DGRI) daher ein Modell, das demjenigen der Finanzierung von Zeitschriften durch Wissenschaftsgesellschaften nachempfunden war. Nach diesem Modell übernimmt die DGRI als gemeinnützige wissenschaftliche Gesellschaft die Finanzierung und eröffnet damit im Gegenzug Möglichkeiten einer engeren Zusammenarbeit. Diese kann darin bestehen, dass JIPITEC für einen internationalen Teil der Veranstaltungen der DGRI sorgt, wie umgekehrt die DGRI die Zeitschrift zur Bewerbung ihrer Veranstaltungen nutzen kann. Angedacht, bislang jedoch nicht verwirklicht, war auch, das Jahrbuch der DGRI als Sonderheft der JIPITEC herauszugeben und die DGRI auf diese Weise von dessen Publikationskosten zu entlasten. Augenblicklich werden verstärkt Anstrengungen unternommen, die Finanzierungslast von JIPITEC entsprechend deren europäischen Charakter auf die Schultern mehrerer nationaler europäischer Computerrechtsgesellschaften (Computer Law Associations, CLAs) zu verteilen.

\section{G. Statistiken}

Die Statistiken wurden um eine Reihe von Faktoren, wie etwa durch Bots verursachten Traffic, bereinigt. Umgekehrt werden durch die „do not track“-Funktion manche Besucher der Seite nicht erfasst. So ist eine genaue Zuordnung nicht immer möglich. Insbesondere lässt sich zwar die Zahl der verschiedenen Besucher ermitteln, dies ist jedoch über die tägliche Statistik hinaus nicht immer aussagekräftig. Weiterhin basieren die Statistiken auf den Log-Files der Server. Die tatsächlichen Werte können teilweise erheblich abweichen. Die Statistiken lassen jedoch klare Trends erkennen. 
Entscheidend erscheint jedoch, dass sich der Trend zu steigenden Besucherzahlen bislang kontinuierlich fortgesetzt hat:

\section{BESUCHE DER WEBSITE}

$\neg$ Besuche

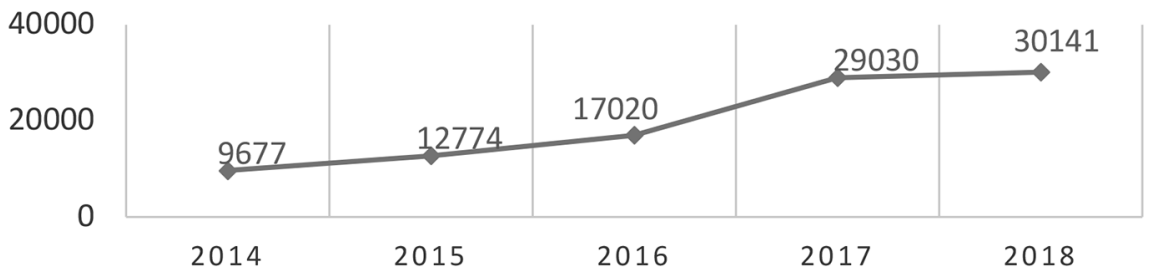

Die Herkunft der Leser ist höchst unterschiedlich, wobei erstaunlich ist, dass fast die Hälfte der Leser aus den USA stammt, was sich gerade in den letzten Jahren entwickelt hat.

\section{6}

17.020 Besuche

\begin{tabular}{|l|c|}
\hline USA & 7.712 \\
\hline Deutschland & 1.767 \\
\hline UK & 1.488 \\
\hline Spanien & 585 \\
\hline Polen & 566 \\
\hline Niederlande & 556 \\
\hline Frankreich & 550 \\
\hline Italien & 436 \\
\hline Russland & 374 \\
\hline China & 312 \\
\hline
\end{tabular}

\section{7}

29.000 Besuche

\begin{tabular}{|l|c|}
\hline USA & 13.457 \\
\hline UK & 3.223 \\
\hline Deutschland & 2.261 \\
\hline Niederlande & 994 \\
\hline Frankreich & 921 \\
\hline Russland & 890 \\
\hline
\end{tabular}


2017

29.000 Besuche

\begin{tabular}{|l|l|}
\hline Polen & 711 \\
\hline Spanien & 689 \\
\hline Italien & 678 \\
\hline China & 431 \\
\hline
\end{tabular}

2018

30.141 Besuche

\begin{tabular}{|l|c|}
\hline USA & 13.961 \\
\hline UK & 3.911 \\
\hline Deutschland & 2.130 \\
\hline Niederlande & 1.023 \\
\hline Frankreich & 1.004 \\
\hline Italien & 936 \\
\hline Spanien & 791 \\
\hline Russland & 677 \\
\hline China & 542 \\
\hline Polen & 516 \\
\hline
\end{tabular}

Nur informationshalber seien hier noch kurz die Artikel genannt, die die höchsten Zugriffszahlen aufweisen:

\begin{tabular}{|l|c|}
\hline Titel & $\begin{array}{l}\text { Page- } \\
\text { views }\end{array}$ \\
\hline $\begin{array}{l}\text { Damian Clifford, EU Data Protection Law and Targeted Advertising: Consent } \\
\text { and the Cookie Monster - Tracking the crumbs of online user behavior }\end{array}$ & 6.465 \\
\hline $\begin{array}{l}\text { Gerald Spindler and Philipp Schmechel, Personal Data and Encryption in the } \\
\text { European General Data Protection Regulation }\end{array}$ & 6.148 \\
\hline $\begin{array}{l}\text { Bart van der Sloot, Ten Questions for Future Regulation of Big Data: A Com- } \\
\text { parative and Empirical Legal Study }\end{array}$ & 5.771 \\
\hline $\begin{array}{l}\text { Brendan Van Alsenoy, Liability under EU Data Protection Law: From Direc- } \\
\text { tive } 95 / 46 \text { to the General Data Protection Regulation }\end{array}$ & 3.669 \\
\hline $\begin{array}{l}\text { Bart van der Sloot, Welcome to the Jungle: The Liability of Internet Interme- } \\
\text { diaries for Privacy Violations in Europe }\end{array}$ & 2.946 \\
\hline $\begin{array}{l}\text { Rolf H. Weber, The Right to Be Forgotten More Than a Pandora's Box? } \\
\text { Rita Matulionyte, Enforcing Copyright Infringements Online: In Search of Ba- } \\
\text { lanced Private International Law Rules }\end{array}$ & 2.937 \\
\hline
\end{tabular}




\section{H. Fazit}

Zehn Jahre JIPITEC haben gezeigt, dass sich diese Open Access Online-Zeitschrift als eine der wenigen juristischen Open-Access-Zeitschriften in Europa etabliert hat. Dafür sprechen zum einen die konstant hohen Zugriffszahlen und zum anderen die vielen veröffentlichten Artikel von renommierten Rechtswissenschaftlern, gerade auch auf internationalem Niveau.

In den juristischen Fachkreisen wird JIPITEC positiv aufgenommen. Dies zeigte sich zum einen in der steigenden Bereitschaft in- wie ausländischer Autoren, vor allem der jüngeren, nachwachsenden Akademikergeneration, ihre Aufsätze in JIPITEC zu veröffentlichen. Dies ist insbesondere mit Blick auf solche Autoren beachtlich, die erstmals in einer reinen Online-Publikation veröffentlichen. Zugleich hat sich die Leserschaft nicht lediglich europäisiert, sondern darüber hinaus auch internationalisiert. So sind besonders viele Besucher aus Staaten der Europäischen Union zu verzeichnen, vor allem insbesondere aus Großbritannien, Frankreich und Spanien. Die meisten Zugriffe kamen darüber hinaus aus den USA, die somit sogar noch vor der Zahl der Zugriffe aus Deutschland rangieren. Aber auch Zugriffe aus Russland, Kanada, China und einer Reihe weiterer Staaten sind zu verzeichnen. Und schließlich wird JIPITEC mittlerweile regelmäßig in konventionellen rechtswissenschaftlichen Zeitschriften und Kommentaren zitiert. ${ }^{8}$

8 Eine Suche nach „JIPITEC“ bei Beck-Online weist immerhin 159 Treffer auf (Abfrage: 13.2.2019). 